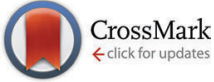

Cite this: J. Mater. Chem. C, 2016 4, 9227

Received 15th July 2016

Accepted 2nd September 2016

DOI: $10.1039 / c 6 t c 02990 f$

www.rsc.org/MaterialsC

\section{Emission stability and reversibility of upconversion nanocrystals $\dagger$}

\author{
Deming Liu, ${ }^{a b c}$ Xiaoxue Xu, ${ }^{\text {abc }}$ Fan Wang, ${ }^{\text {abc }}$ Jiajia Zhou, ${ }^{c d}$ Chao Mi, ${ }^{\text {bc }}$ Lixin Zhang, ${ }^{a}$ \\ Yiqing Lu, ${ }^{a}$ Chenshuo Ma, ${ }^{a}$ Ewa Goldys, ${ }^{a}$ Jun Lin ${ }^{c e}$ and Dayong Jin ${ }^{a a b c}$
}

\begin{abstract}
Rare-earth doped upconversion nanocrystals have emerged as a novel class of luminescent probes for biomedical applications. The knowledge about their optical stability in aqueous solution under different $\mathrm{pH}$ and temperature conditions has not been comprehensively explored. Here we conduct a systematic investigation and report the emission stability and reversibility of typical $\mathrm{NaYF}_{4}: \mathrm{Yb}^{3+}, \mathrm{Er}^{3+}$ nanocrystals and their core-shell nanostructures in aqueous solution at different temperatures and with different $\mathrm{pH}$ values. These nanocrystals show reversible luminescence response to temperature changes, while low $\mathrm{pH}$ permanently quenches their luminescence. With the addition of inert shells, with thicknesses ranging from $1.5 \mathrm{~nm}$ to $8 \mathrm{~nm}$, the emission stability and reversibility change significantly. Thicker inert shells not only lead to a significant enhancement in the emission intensity but also stabilize its optical responses which become less affected by temperature variations and $\mathrm{pH}$ conditions. This study suggests that upconversion nanocrystal-based sensitive temperature and $\mathrm{pH}$ sensors do not generally benefit from the core-shell structure usually recommended for enhanced upconversion luminescence.
\end{abstract}

\section{Introduction}

Lanthanide-doped upconversion nanoparticles (UCNPs) that absorb near infrared excitation and exhibit anti-Stokes emission in visible and ultra-violet regions have attracted significant interest in the last decade. ${ }^{1,2}$ They offer key advantages in bioimaging and luminescence detection including low detection background, tunable emission spectrum ${ }^{3}$ and decay lifetime, ${ }^{4,5}$ the absence of photo-bleaching and blinking, ${ }^{6,7}$ deep penetration of the excitation light, ${ }^{8}$ and low toxicity with excellent chemical and biological compatibility. ${ }^{9,10}$ They are widely regarded as ideal luminescent probes for a range of biomedical applications, such as ultrasensitive biomolecular assays, ${ }^{11-14}$ multimodal biomedical imaging, ${ }^{15-21}$ and photodynamic therapies. ${ }^{22,23}$

\footnotetext{
${ }^{a}$ Advanced Cytometry Labs, ARC Centre of Excellence for Nanoscale BioPhotonics, Macquarie University, Sydney, NSW, 2109, Australia.

E-mail: xiaoxue.xu@mq.edu.au,dayong:jin@uts.edu.au

${ }^{b}$ Institute for Biomedical Materials and Devices, Faculty of Science, University of Technology Sydney, NSW, 2007, Australia

${ }^{c}$ Australian Research Council Research Hub for Integrated Device for End-user Analysis at Low-levels (IDEAL), Faculty of Science, University of Technology Sydney, NSW, 2007, Australia

${ }^{d}$ College of Optical Science and Engineering, State Key Laboratory of Modern Optical Instrumentation, Zhejiang University, Hangzhou, 310027, P. R. China ${ }^{e}$ State Key Laboratory of Rare Earth Resource Utilization, Changchun Institute of Applied Chemistry, Chinese Academy of Sciences, Changchun, 130022, P. R. China

$\dagger$ Electronic supplementary information (ESI) available. See DOI: 10.1039/c6tc02990f
}

Several groups reported that UCNPs can serve as accurate nanoscale sensors for intracellular monitoring of temperature ${ }^{24}$ or $\mathrm{pH}^{25}$ They can also be applied as photoresponsive carriers for controlled delivery and release of drugs in vivo, ${ }^{26-31}$ where high temperature and/or lower $\mathrm{pH}$ conditions trigger drug release. The ratio-metric green emissions of $\mathrm{Er}^{3+}$ doped UCNPs are sensitive to cellular temperature with a resolution of $0.5{ }^{\circ} \mathrm{C}$ in the biological range of $25{ }^{\circ} \mathrm{C}$ to $45{ }^{\circ} \mathrm{C} .{ }^{32}$ The red emission of the $\mathrm{Er}^{3+}$ UCNPs was found to be responsive to $\mathrm{pH}$ variations and to increase at low $\mathrm{pH}^{32} \mathrm{Li}$ and co-workers recently reported a hybrid design of carbon@ inert shell @UCNPs for real-time monitoring of the localized temperature increase during photodynamic therapy. ${ }^{33}$ We demonstrated a drug release sensing scheme by using the luminescence intensity of UCNPs. In this scheme we monitored the drug release process in the low $\mathrm{pH}$ environment (around $\mathrm{pH}=5$ ), as the drug on the surface of UCNPs was designed to quench the luminescence increase upon drug release. ${ }^{34}$

The understanding of luminescence responses to the varied temperature and $\mathrm{pH}$ environment ${ }^{35-37}$ require more detailed consideration of the stability and reversibility of UCNP luminescence under different $\mathrm{pH}$ and temperature conditions. The literature reports on the subject remain scattered. Wei et al. reported the stable emission of the UC nanocrystals with different surface ligands, but it was only tested at neutral $\mathrm{pH}$ in aqueous solutions. ${ }^{38} \mathrm{Li}$ et al. reported the thermal stability of UCNPs and an anomalous relationship between temperature and the upconversion luminescence. ${ }^{39,40} \mathrm{Xu}$ et al. reported that 
$\alpha-\mathrm{NaYb}(\mathrm{Mn}) \mathrm{F}_{4}: \mathrm{Er}^{3+} @ \mathrm{NaYF}_{4}$ UCNPs can be applied as luminescent nano-thermometers over a wide temperature range. But these tests were done only for the dry powder.

Coating inert shells onto the core UCNPs is a wellestablished and effective solution to minimize the surface quenching effect and therefore to enhance the upconversion luminescence by a factor of several to hundreds, depending on the core size and shell thickness. ${ }^{41-44}$ However, the role of the inert shells in influencing the stability and reversibility of luminescence at varied $\mathrm{pH}$ and temperature in aqueous solution has not yet been investigated.

In this work, we systematically investigated and compared the luminescence response of $\mathrm{Er}^{3+}$ doped UCNPs to different $\mathrm{pH}$ and temperature values in aqueous solution for both coreonly and core-shell UCNPs. Using a modified hot-injection method, we prepared a systematic series of UCNPs by epitaxial growth of the homogeneous $\mathrm{NaYF}_{4}: \mathrm{Yb}^{3+}, \mathrm{Er}^{3+}$ UCNPs with different shell thicknesses from 1.5 to $8 \mathrm{~nm}$. To characterize these nanoparticles in aqueous solution, we removed the surface organic ligand of oleic acids and transfer the hydrophilic UCNPs into the aqueous phase using an acid-based ligand removal method reported by Capobianco et $a{ }^{45}$ We chose this approach to reduce the influence of surface ligands ${ }^{38}$ on UCNP upconversion luminescence. Our results show that the luminescence intensity of UCNPs decreases in either an acid or an alkali pH environment. In an acidic environment this luminescence decrease is not reversible. Conversely, higher temperature significantly reduces the luminescence intensity in a reversible way. The inert shell also reduces the sensitivity and resolution of the ratiometric response of green emissions $(524 \mathrm{~nm} / 545 \mathrm{~nm})$ of $\mathrm{Er}^{3+}$ doped UCNPs for temperature sensing below $60{ }^{\circ} \mathrm{C}$. This work compliments the current knowledge underpinning the rapid development of upconversion nanosensors for emerging biomedical applications and further suggests that new careful designs are necessary to accurately sense temperature and $\mathrm{pH}$ in specific applications.

\section{Materials and methods}

Yttrium chloride hexahydrate $\left(\mathrm{YCl}_{3} \cdot 6 \mathrm{H}_{2} \mathrm{O}, 99.99 \%\right)$, ytterbium chloride hexahydrate $\left(\mathrm{YbCl}_{3} \cdot 6 \mathrm{H}_{2} \mathrm{O}, 99.998 \%\right)$, erbium chloride hexahydrate $\left(\mathrm{ErCl}_{3} \cdot 6 \mathrm{H}_{2} \mathrm{O}, 99.9 \%\right)$, sodium hydroxide $(\mathrm{NaOH}, 98 \%)$, ammonium fluoride $\left(\mathrm{NH}_{4} \mathrm{~F}, 99.99 \%\right)$, oleic acid (OA, 90\%), 1-octadecene (ODE, 90\%) and hydrochloric acid ( $\mathrm{HCl}, 37 \%)$ were purchased from Sigma-Aldrich. Oleylamine (OM, 90\%) was purchased from Pfaltz \& Bauer. All reagents were used as received without further purification.

\section{Synthesis of $\beta-\mathrm{NaYF}_{4}: \mathrm{Yb}^{3+}, \mathrm{Er}^{3+}$ cores}

A modified synthesis method was adopted to prepare $\beta-\mathrm{NaYF}_{4}: 20 \% \mathrm{Yb}^{3+}, 2 \% \mathrm{Er}^{3+}$ as core UCNPs. In a typical procedure, $1 \mathrm{~mL}$ of $\mathrm{LnCl}_{3}$ in methanol (1.0 mmol, $\left.\mathrm{Ln}=\mathrm{Y}, \mathrm{Yb}, \mathrm{Er}\right)$ was mixed with OA $(6 \mathrm{~mL})$ and ODE $(15 \mathrm{~mL})$ in a $100 \mathrm{~mL}$ three-neck roundbottom flask. The mixture solution was degassed under Ar flow during the heating up to $150{ }^{\circ} \mathrm{C}$ followed by $30 \mathrm{~min}$ isothermal reaction to form a clear solution, and then cooled down to room temperature. $10 \mathrm{~mL}$ of methanol containing $\mathrm{NH}_{4} \mathrm{~F}(4 \mathrm{mmol})$ and $\mathrm{NaOH}(2.5 \mathrm{mmol})$ was added to the flask and then stirred for $60 \mathrm{~min}$. The solution was slowly heated up to $110{ }^{\circ} \mathrm{C}$ and kept at $110{ }^{\circ} \mathrm{C}$ for $30 \mathrm{~min}$ to completely remove the methanol and any residual water. Then the reaction solution was quickly heated up to $310{ }^{\circ} \mathrm{C}$ and kept isothermally for $1 \mathrm{~h}$, before being cooled down to room temperature. Ethanol was added to precipitate the nanocrystals, which were washed 4 times with cyclohexane, ethanol and methanol. The obtained pure $\mathrm{NaYF}_{4}: \mathrm{Yb}^{3+}, \mathrm{Er}^{3+}$ nanocrystals were re-dispersed in $10 \mathrm{~mL}$ of cyclohexane for coating the inert shell.

\section{Synthesis of $\alpha-\mathrm{NaYF}_{4}$ shell precursors}

A modified literature method ${ }^{46}$ was used, with all the preparation and pre-treatment steps similar to that in the $\mathrm{NaYF}_{4}: \mathrm{Yb}^{3+}, \mathrm{Er}^{3+}$ core synthesis method. The only two differences were that the lanthanide precursor was changed to be $1 \mathrm{mmol} \mathrm{YCl}_{3}$ and the reaction temperature became $290{ }^{\circ} \mathrm{C}$ (instead of $310{ }^{\circ} \mathrm{C}$ ). After the reaction, the nanoparticles were washed and re-dispersed in cyclohexane. The obtained pure $\alpha-\mathrm{NaYF}_{4}$ seeds in cyclohexane solution was mixed with $10 \mathrm{~mL}$ of ODE in one $100 \mathrm{~mL}$ threeneck flask. Then, the mixture was kept at $110{ }^{\circ} \mathrm{C}$ for $30 \mathrm{~min}$ under Ar flow. After the mixture was cooled to room temperature, $\alpha-\mathrm{NaYF}_{4}$ ODE solution as the precursor of the inert shell was obtained.

\section{Synthesis of $\beta-\mathrm{NaYF}_{4}: \mathrm{Yb}^{3+}, \mathrm{Er}^{3+} @ \mathrm{NaYF}_{4}$ core-shell nanocrystals}

A modified hot-injection method ${ }^{46}$ was used for growing undoped shells onto the core nanocrystals. $1 \mathrm{~mL}\left(0.2 \mathrm{mmol} \mathrm{Ln}^{3+}\right)$ the obtained $\mathrm{NaYF}_{4}: \mathrm{Yb}^{3+}, \mathrm{Er}^{3+}$ core cyclohexane stock solution was mixed with OA $(5 \mathrm{~mL}), \mathrm{OM}(1 \mathrm{~mL})$ and $\mathrm{ODE}(8 \mathrm{~mL})$ in a $100 \mathrm{~mL}$ three-neck flask. The mixture was degassed under Ar flow and kept at $110{ }^{\circ} \mathrm{C}$ for $30 \mathrm{~min}$ to completely remove cyclohexane as well as any residual water. After that, it was quickly heated to $305{ }^{\circ} \mathrm{C}$ and the pure $\alpha-\mathrm{NaYF}_{4}$ seeds in ODE solution were injected using a syringe (injection rate: $0.05 \mathrm{mmol} \alpha-\mathrm{NaYF}_{4}$ seed solution every $10 \mathrm{~min}$; for total amounts of injected $\alpha-\mathrm{NaYF}_{4}$ seeds for shells of different thicknesses refer to Table S1, ESI $\dagger$ ). After the reaction, the precipitate was washed and stored in cyclohexane.

\section{Characterization}

Standard transmission electron microscope (TEM) measurements were performed using a Philips CM10 TEM equipped with an Olympus Sis Megaview G2 Digital Camera. The samples were prepared for TEM analysis by placing a drop of a dilute suspension of nanocrystals onto formvar-coated copper grids (300 meshes) and were allowed to dry in a desiccator at room temperature before use. Powder X-ray diffraction (XRD) patterns were obtained on a PANalytical X'Pert Pro MPD X-ray diffractometer using $\mathrm{Cu}$ Ka1 radiation $(40 \mathrm{kV}, 40 \mathrm{~mA}, \lambda=0.15418 \mathrm{~nm})$. The XRD samples were prepared by repeatedly drying drops of nanocrystal dispersions in cyclohexane cast on a zerobackground silicon wafer. The temperature-dependent and $\mathrm{pH}$ dependent upconversion luminescence spectra of colloidal 
solutions in quartz cuvettes with $10 \mathrm{~mm}$ path length were acquired using a Fluorolog-Tau3 spectrofluorometer (JobinYvonHoriba) equipped with an external $980 \mathrm{~nm}$ CW diode laser with a pump power of $200 \mathrm{~mW}$. UCNPs in the aqueous dispersion were tuned to the same number concentration. Temperature control is via a homemade water bath equipment. The $\mathrm{pH}$ values of UCNPs dispersed in aqueous solution were adjusted using Milli-Q water and the PBS solutions with $\mathrm{pH} 3$ and 12 respectively. The luminescence lifetimes were measured using a purpose-built high-throughput 3-dimensional time-resolved spectrometer, ${ }^{47}$ with pulsed $980 \mathrm{~nm}$ laser excitation $(100 \mathrm{~mW})$ at a repetition rate of $50 \mathrm{~Hz}$.

\section{Results and discussion}

Fig. 1 illustrates our design of the experiments to systematically study the luminescence response of core-shell UCNPs in aqueous solution in varying $\mathrm{pH}$ and temperature environments. Homogeneous core-shell structures were synthesized on the $\mathrm{NaYF}_{4}: \mathrm{Yb}^{3+}, \mathrm{Er}^{3+}$ core UCNPs by controlling the amount of OM in a hot injection method (Fig. S1, ESI $\dagger$ ). The shell thickness of homogeneous core-shell UCNPs was controlled by adjusting the amount of shell precursors. (Table S1, ESI $\dagger$ ). Fig. S2 (ESI $\dagger$ ) showed that the $24 \mathrm{~nm} \beta-\mathrm{NaYF}_{4}: \mathrm{Yb}^{3+}, \mathrm{Er}^{3+}$ core was sequentially coated with a series of $\mathrm{NaYF}_{4}$ shells of different thicknesses. These samples were treated with a diluted acid solution to remove the surface ligands so that the as-prepared UCNPS became hydrophilic and were transferred into aqueous solution (Fig. 1a). Such ligand-free UCNPs will avoid the test influence from surface ligands. Fig. 1b-k shows the morphology characterization of the as-prepared hydrophilic core and core-shell UCNP samples. $\mathrm{NaYF}_{4}: \mathrm{Yb}^{3+}, \mathrm{Er}^{3+}$ core UCNPs displayed uniform spherical shape, with an average size of $24.1 \mathrm{~nm}$ (Fig. 1b and g) and a narrow size distribution. The core-shell nanocrystals were also spherical, with average sizes of $27.2 \mathrm{~nm}, 28.9 \mathrm{~nm}$, $33.2 \mathrm{~nm}$ and $40.2 \mathrm{~nm}$, respectively, and have narrow size distribution (Fig. 1c-f). Their average shell thickness was calculated by comparing the average diameters of core and core-shell nanocrystals ${ }^{48}$ from Fig. $1(\mathrm{~g}-\mathrm{k})$.

Fig. 2(a) illustrates the major four emissions' energy transfer processes between energy levels of $\mathrm{Yb}^{3+}$ and $\mathrm{Er}^{3+}$ under $980 \mathrm{~nm}$ light excitation. The three-dimensional time resolved luminescence spectrum of UCNPs was obtained by high-throughput time-resolved luminescence spectroscopy that simultaneously provides the emission spectrum and the lifetime decay curve, as shown in Fig. 2(b). The luminescence enhancement and increased lifetimes due to coating of the UCNPs with the inert (a)
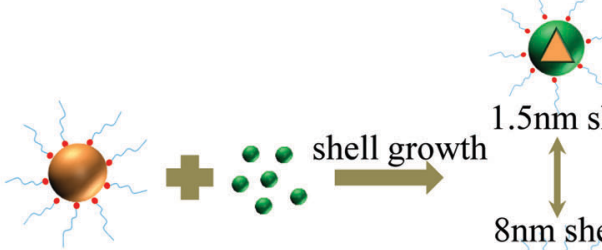

$1.5 \mathrm{~nm}$ shell

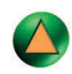

Remove

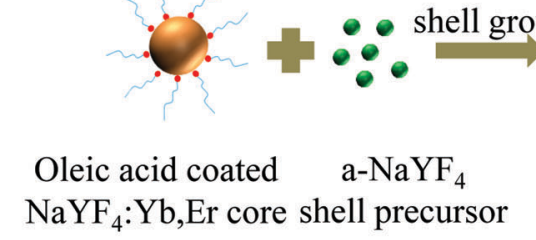

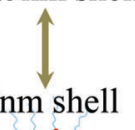

oleic acid

$8 n m$ shell

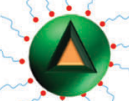

Oleic acid coated

$\mathrm{NaYF}_{4}$ core-shell

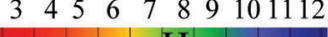

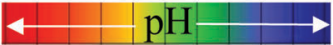

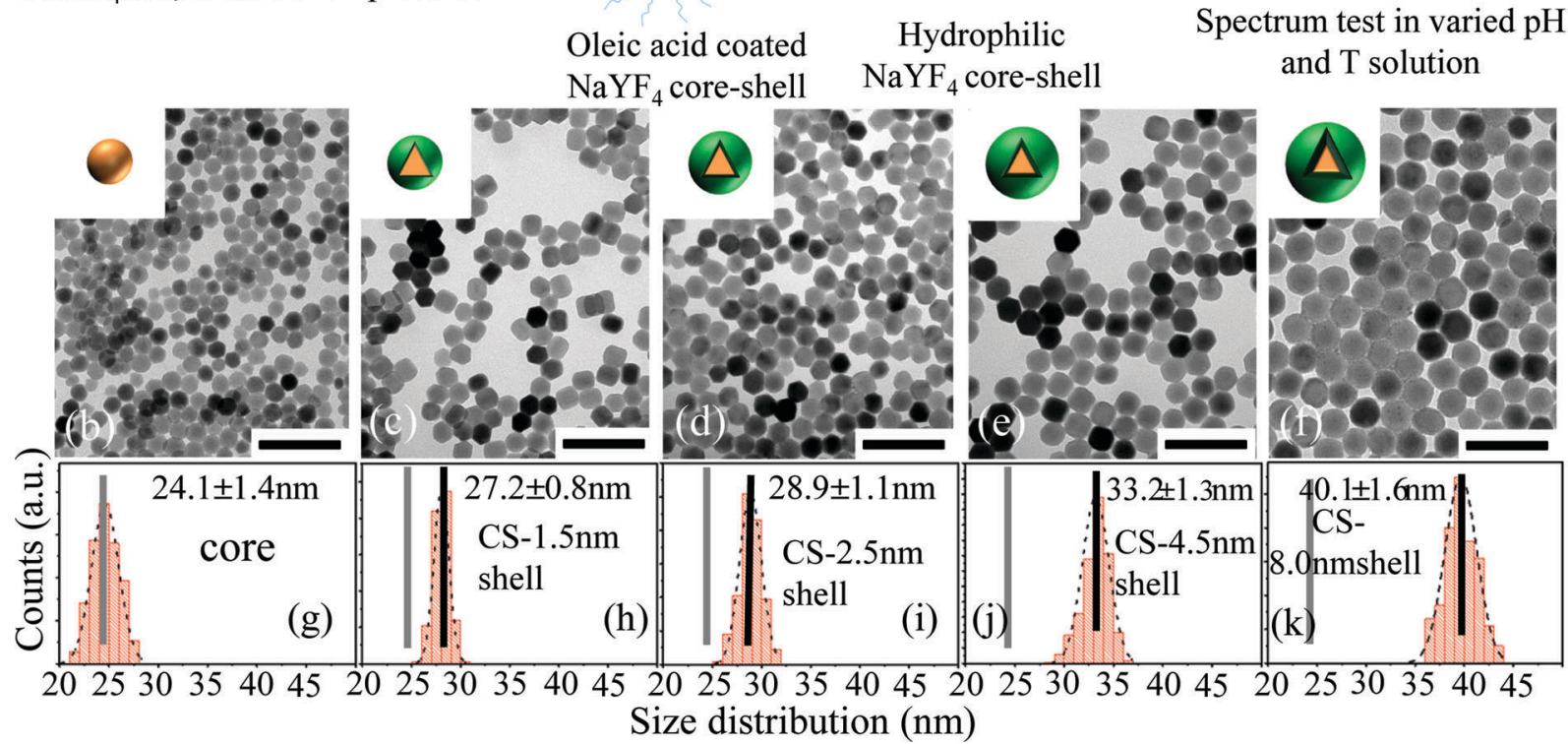

Fig. 1 Synthesis and testing of the core and core-shell UCNPs' luminescence response to varying $\mathrm{pH}$ and temperature environments (a), TEM images and size distribution charts of core-only (b, g) and core-shell UCNPs with $1.5 \mathrm{~nm}(\mathrm{c}, \mathrm{h}), 2.5 \mathrm{~nm}(\mathrm{~d}, \mathrm{i}), 4.5 \mathrm{~nm}(\mathrm{e}, \mathrm{j})$ and $8.0 \mathrm{~nm}(\mathrm{f}, \mathrm{k})$ homogeneous NaYF 4 shells. (Scale bar: $50 \mathrm{~nm}$ ). 


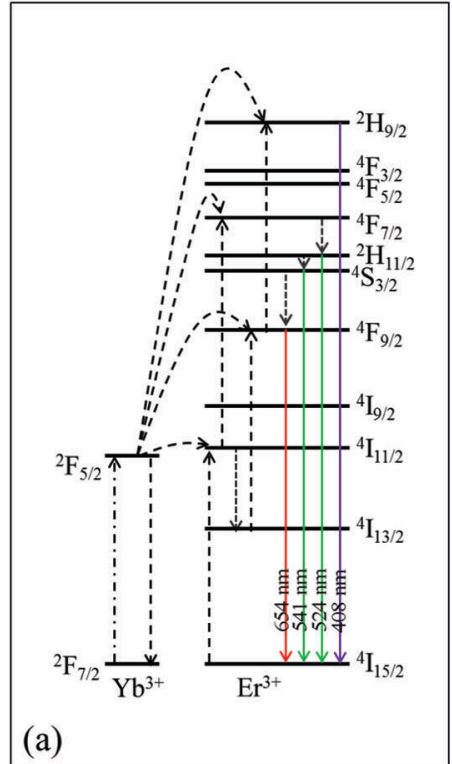

(c)

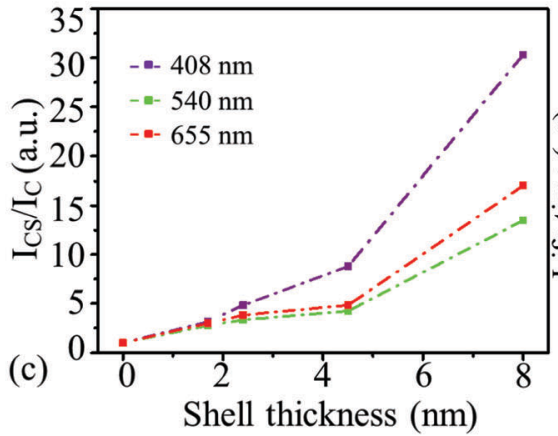

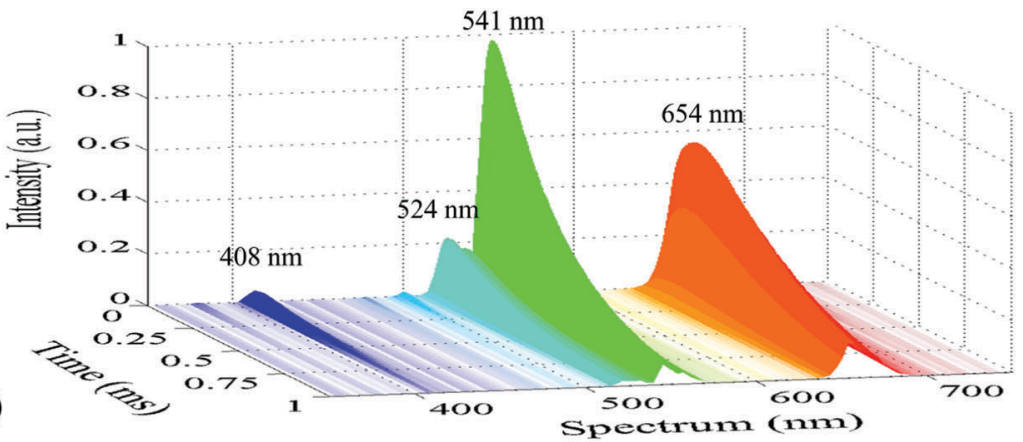

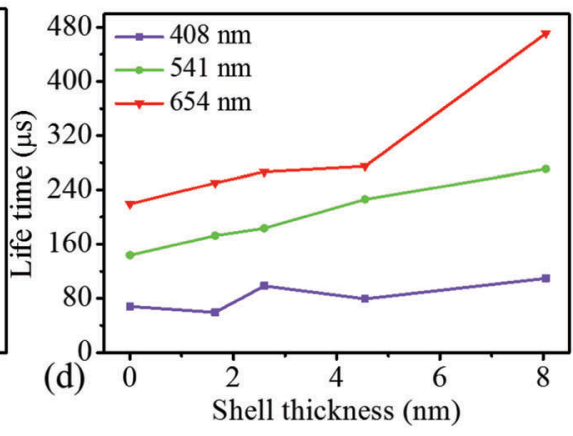

Fig. 2 (a) Schematic illustration of the transition energy levels of $\mathrm{NaYF}_{4}: \mathrm{Yb}^{3+}, \mathrm{Er}^{3+}$ nanocrystals. Arrows indicate: $-\cdot \rightarrow \mathrm{NIR}$ excitation, $--\rightarrow$ energy transfers, $--\rightarrow$ multiphonon nonradiative relaxation, $\longrightarrow, \longrightarrow, \longrightarrow$ and upconversion emissions. (b) Typical real-time resolved emission spectrum of $\mathrm{NaYF}_{4}: \mathrm{Yb}^{3+}, \mathrm{Er}^{3+}$ UCNPs. Intensity enhancement factors (c) and luminescence decay lifetimes (d) for emissions at $408 \mathrm{~nm}, 540 \mathrm{~nm}$ and $655 \mathrm{~nm}$ were plotted as functions of shell thickness.

shells (as shown in Fig. S3 and S4, ESI $\dagger$ ) was consistent with other reports in the literature. ${ }^{43,46,49-51}$ Fig. 2(c) shows that the enhancement of emissions increased with increased thickness of the shells, while enhancement factors at varied emission positions were also different. The emission peaks at $408 \mathrm{~nm}$, $540 \mathrm{~nm}$, and $655 \mathrm{~nm}$ were only enhanced by about 3 times by coating a $1.5 \mathrm{~nm}$ inert $\mathrm{NaYF}_{4}$ shell, and the enhancement factors of 30,12 and 15 were achieved for these peaks when a $8 \mathrm{~nm}$ shell was coated. Similar exceptional enhancement of violet emissions was also observed for the $\mathrm{NaYF}_{4}: \mathrm{Yb}^{3+}, \mathrm{Tm}^{3+}$ @ $\mathrm{CaF}_{2}$ core-shell nanocrystals. ${ }^{52}$ This suggested that higher order energy transfer processes benefited more from the inert shell protection. The luminescence lifetime data, shown in Fig. 2(d), further confirmed that the uniform shells were coated onto the core UCNPs. The luminescence lifetimes from the three emission bands (violet, green and red) increased with thicker shells. Both these strongly enhanced emission intensities and significantly longer lifetimes confirmed the successful growth of a series of homogeneous core-shell UCNPs.

Fig. 3 and Fig. S5 (ESI $\dagger$ ) show the impact of the shell on the luminescence stability and reversibility against $\mathrm{pH}$ changes. The luminescence spectra of core-only and core-shell UCNPs were tested in aqueous solution under varying $\mathrm{pH}$ values. Fig. S5 (ESI $\dagger$ ) shows that the acidic solution had a strong quenching effect on the UCNPs' luminescence and the inert shells were able to alleviate the quenching to some degree. Both the green and red emissions displayed similar emission quenching trends with the $\mathrm{pH}$ decrease (Fig. 3(a)). The green emission from the core UCNPs decreased to $28 \%$ of its initial intensity with the $\mathrm{pH}$ decreasing from 7 to 3 , while the green emission from the core-shell UCNPs only decreased to about $65 \%$ of its original intensity. The alkaline conditions have been found to have less influence on the emissions. The emission from the core UCNPs was reduced only by $20 \%$ with the $\mathrm{pH}$ increasing from 7 to 12 , while the emission from core-shell UCNPs only experienced a slight decrease under alkaline conditions. These data showed that the inert shells help in improving the luminescence stability against $\mathrm{pH}$ changes.

Fig. 3(b) shows the luminescence reversibility of luminescence of the core and core-shell UCNPs when repeatedly varying the $\mathrm{pH}$ conditions between $\mathrm{pH}=4$ and $\mathrm{pH}=7$. The quenching effect by the acidic aqueous solution was not reversible regardless of the presence of thick inert shells. Once the $\mathrm{pH}$ of the samples was decreased to $\mathrm{pH}=4$ for less than ten minutes, irreversible luminescence quenching occurred. Luminescence in these samples did not recover even by bringing the pH back to 7 . The irreversible luminescence quenching of the UNCPs in the acidic environment suggested that the crystal surface may experience chemical damage under low $\mathrm{pH}$ conditions. To prove this, we carefully compared the TEM images of ligand free UCNPs before (Fig. 3(c)) and after (Fig. 3(d)) treatment with the $\mathrm{pH}=3$ acid solution for 1 hour. Interestingly, we observed an obvious decrease in size from $24 \mathrm{~nm}$ to $21 \mathrm{~nm}$ and a rough surface morphology, showing that the nanocrystals were chemically etched. This chemical damage from weak acid was also found in other rare earth fluoride nanocrystals, such as $\mathrm{NaEuF}_{4}{ }^{53}$ and $\mathrm{Lu}_{6} \mathrm{O}_{5} \mathrm{~F}_{8} \cdot{ }^{54}$ We attributed this to the dissociation of Ln-F bonds of the $\mathrm{NaYF}_{4}$ nanocrystals in the solution with low $\mathrm{pH}(\leq 3)$. 

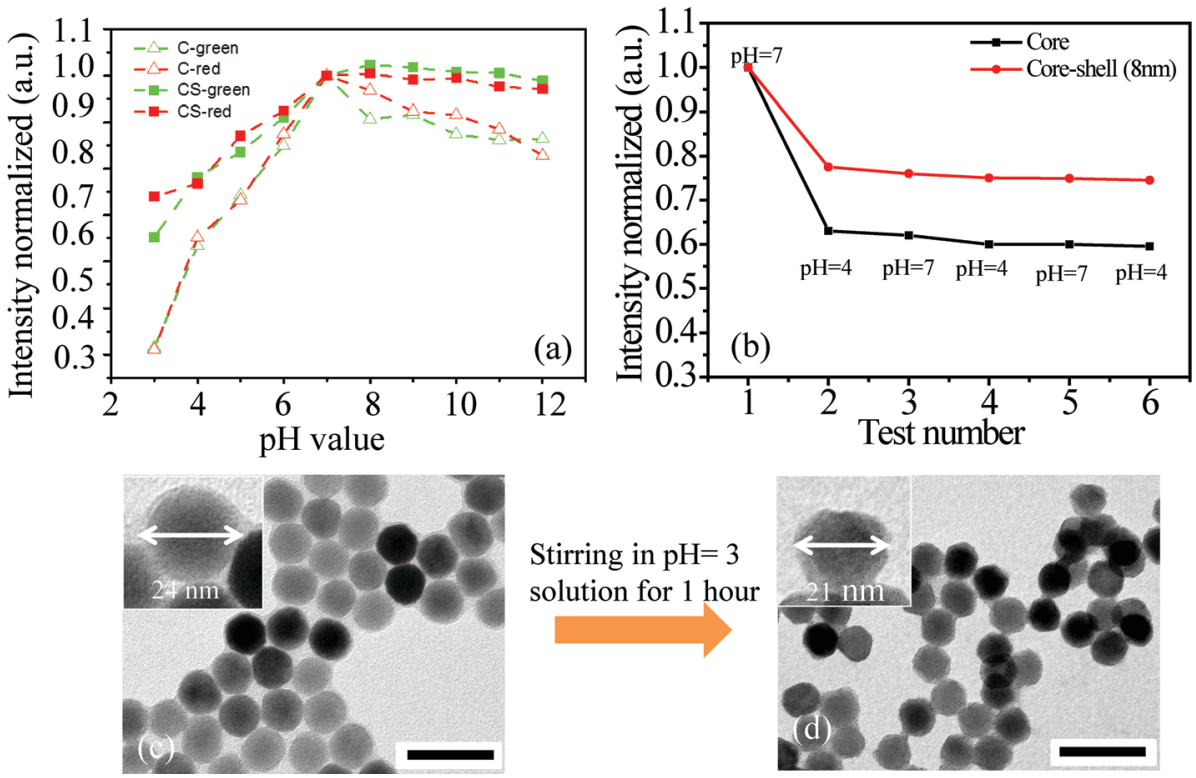

Fig. 3 (a) Luminescence responses of the $\mathrm{NaYF}_{4}: \mathrm{Yb}^{3+}, \mathrm{Er}^{3+}$ core UCNPs and $\mathrm{NaYF}_{4}: \mathrm{Yb}^{3+}, \mathrm{Er}^{3+} \mathrm{aNaYF}_{4}$ core-shell UCNPs in the green $(524 \mathrm{~nm}$ and $545 \mathrm{~nm}$ ) and red emission bands. The data were normalized at $\mathrm{pH}=7$ and plotted as a function of $\mathrm{pH}$ values of the solution. (b) The reversibility test for the $655 \mathrm{~nm}$ emissions of the core and core-shell UCNPs by switching the $\mathrm{pH}$ values between $\mathrm{pH}=4$ and $\mathrm{pH}=7$. TEM images of ligand free UCNPs before (c) and after (d) stirring in $\mathrm{pH}=3$ solution for 1 hour. The inset TEM images in (c) and (d) show the size changes of UCNPs before and after the treatment. (Scale bar is $50 \mathrm{~nm}$ ).

We further measured the spectra of $\mathrm{NaYF}_{4}: \mathrm{Yb}^{3+}, \mathrm{Er}^{3+}$ core-only and core-shell UCNPs in Milli-Q water at various temperatures from $10{ }^{\circ} \mathrm{C}$ to $80{ }^{\circ} \mathrm{C}$ (shown in Fig. 4 and Fig. S6, ESI $\dagger$ ). Fig. S6a and $\mathrm{b}$ (ESI $\dagger$ ) shows the luminescence intensities of both core-only and core-shell nanocrystals decreased at a higher temperature with the core UCNPs being more strongly affected. The green emission of the core-only UCNPs decreased quickly to $78 \%$ of its original intensity even when the temperature was increased only from $10{ }^{\circ} \mathrm{C}$ to $30{ }^{\circ} \mathrm{C}$. With a further temperature increase from $30{ }^{\circ} \mathrm{C}$ to $80{ }^{\circ} \mathrm{C}$, the green emission decreased to nearly half of its original intensity at $10{ }^{\circ} \mathrm{C}$. The shell protection again was found to alleviate the temperature-induced quenching, with the green emission from the core-shell UCNPs decreasing by only $27 \%$ at $80^{\circ} \mathrm{C}$. The quenching of the red emission was similar to the green emission, when it occurred at lower temperatures from $10{ }^{\circ} \mathrm{C}$ to
$40{ }^{\circ} \mathrm{C}$. However, the red emission has slightly higher stability than the green emission in the higher temperature range from $40{ }^{\circ} \mathrm{C}$ to $80{ }^{\circ} \mathrm{C}$. We think that the reason for red emission receiving a lower quenching effect than the green emission at the higher temperature range is due to an increase of population transition from ${ }^{4} \mathrm{~S}_{3 / 2}$ to ${ }^{4} \mathrm{~F}_{9 / 2}$. According to Xiaogang Liu group's investigation ${ }^{55}$ on the relationship between relative emission intensity and nanocrystal surface defects that the higher energy level excited state would receive multiphonon nonradioactive relaxation from surface quenchers. The relative low energy level of ${ }^{4} \mathrm{~F}_{9 / 2}$ would receive some population from the higher energy levels $\left(\right.$ e.g. $\left.{ }^{4} \mathrm{~S}_{3 / 2}\right)$, and therefore the red emission presents relatively higher stability than the green emission at a higher temperature.

Fig. 4(b) demonstrates the reversibility of the luminescence emission against temperature, and it shows that the emission
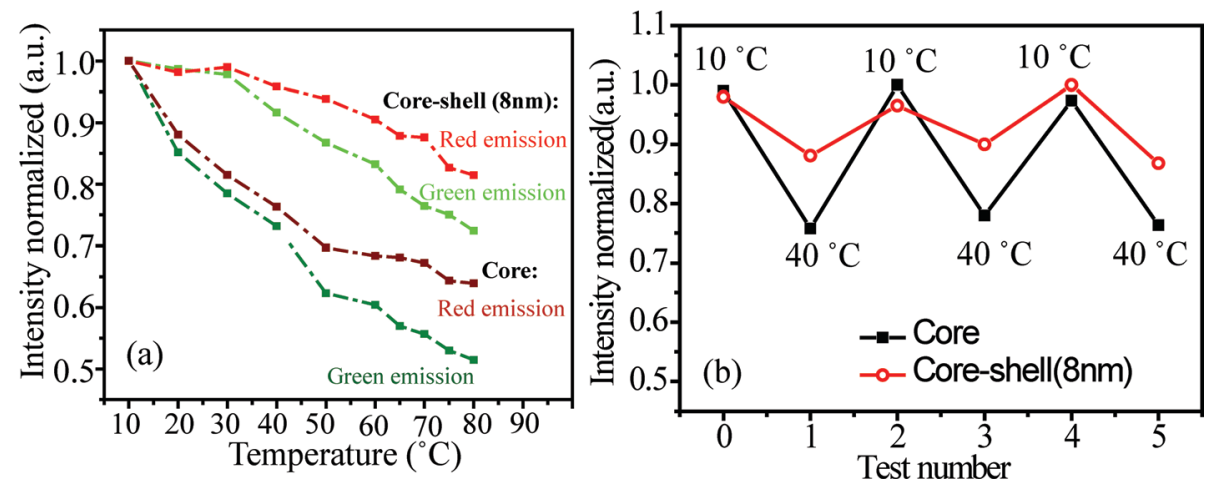

Fig. 4 (a) The intensity response at green and red bands for the core and core-shell UCNPs as a function of temperature. The data were normalized at $10^{\circ} \mathrm{C}$; (b) green emission intensity response as a function of temperature repeatedly switched between $10^{\circ} \mathrm{C}$ and $40{ }^{\circ} \mathrm{C}$. 

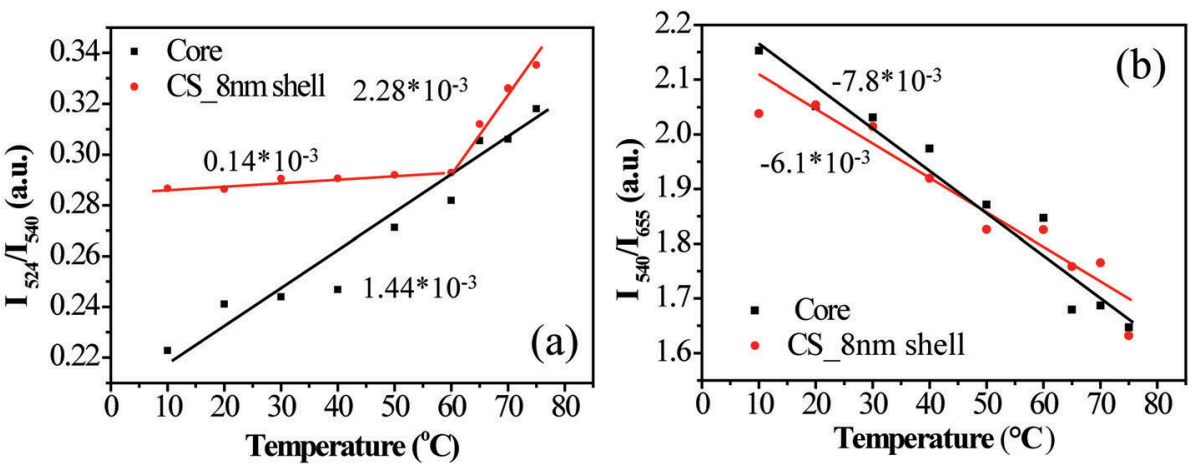

Fig. 5 Emission intensity ratio (a) of $524 \mathrm{~nm} / 540 \mathrm{~nm}$ from core and core-shell UCNPs and (b) emission intensity ratio of $540 \mathrm{~nm} / 655 \mathrm{~nm}$ from core and core-shell UCNPs as a function of temperature.

intensities of both the core-only and core-shell nanocrystals followed the temperature change between $10{ }^{\circ} \mathrm{C}$ and $40{ }^{\circ} \mathrm{C}$, and these changes were fully reversible. The degree of intensity variations for the core-shell nanocrystals was lower than that of the core nanocrystals. These results suggested that the coreshell structure of $\mathrm{NaYF}_{4}: \mathrm{Yb}^{3+}, \mathrm{Er}^{3+} / \mathrm{NaYF}_{4}$ has a less pronounced response to temperature variations, especially in the range from $10{ }^{\circ} \mathrm{C}$ to $40{ }^{\circ} \mathrm{C}$, relevant for in vivo biomedical applications. The observed improvement in emission stability by coating the passivation shell suggested that the inert shell can effectively reduce the quenching effect from the vibrational energies and optical traps arising from the particle surface. The water molecule is known as a surface oscillator that significantly quenches the luminescence of lanthanide dopant ions, because of the high energy $\left(c a .3500 \mathrm{~cm}^{-1}\right)$ of the stretching vibration. As the temperature increases, the water molecular on the crystal surface could be more intensely vibrating, which may affect the Ln-F bond on the crystal surface and create more phonons. By coating the inert shell structure, forming a gap between the emitters and the quenchers can effectively reduce the quenching effect caused by a temperature increase.

The ratiometric emissions $\left(I_{524} / I_{540}\right)$ from the $\mathrm{NaYF}_{4}: \mathrm{Yb}^{3+}, \mathrm{Er}^{3+}$ UCNPs have been broadly used in nanoscale thermometry ${ }^{56}$ because the population distributions on ${ }^{2} \mathrm{H}_{11 / 2}$ and ${ }^{4} \mathrm{~S}_{3 / 2}$ are dominated by Boltzmann's thermal distribution and elevated temperature leads to rapid population of the ${ }^{2} \mathrm{H}_{11 / 2}$ state. ${ }^{57}$ Fig. 5 shows the intensity ratios of $524 \mathrm{~nm}$ to $540 \mathrm{~nm}\left(I_{524} / I_{540}\right)$ and $540 \mathrm{~nm}$ to $655 \mathrm{~nm}\left(I_{540} / I_{655}\right)$ emissions for the core-only and core-shell nanocrystals. The ratio of $I_{524} / I_{540}$ in Fig. 5(a) for the core nanocrystals shows a linear increase with temperature in the range from $10{ }^{\circ} \mathrm{C}$ to $80^{\circ} \mathrm{C}$, which is consistent with previously reported results. ${ }^{57,58}$ The ratio of $I_{524} / I_{540}$ of core-shell nanocrystals is slightly higher than that of core-only nanocrystals at $10{ }^{\circ} \mathrm{C}$, which suggests that the inert shell effectively saved some population on ${ }^{2} \mathrm{H}_{11 / 2}$ from nonradioactive relaxation. As the temperature was increased from $10{ }^{\circ} \mathrm{C}$ to $60{ }^{\circ} \mathrm{C}$, the ratio of $I_{524} / I_{540}$ of core-shell nanocrystals slightly increases; however, the ratio increases quickly when the temperature was increased from $60{ }^{\circ} \mathrm{C}$ to $75{ }^{\circ} \mathrm{C}$. This anomalous temperature-dependent phenomenon was also found and reported by other groups for small sized upconversion nanocrystals. ${ }^{59}$ The population distribution changes on ${ }^{2} \mathrm{H}_{11 / 2}$ and ${ }^{4} \mathrm{~S}_{3 / 2}$ could be caused by surface defects and surface molecular quenchers (e.g. water molecular vibration) and temperature. Higher temperature helps to increase the population distribution on ${ }^{2} \mathrm{H}_{11 / 2}$. On the other hand, stronger surface quenchers would decrease the population on ${ }^{2} \mathrm{H}_{11 / 2}$ compared to that on ${ }^{4} \mathrm{~S}_{3 / 2}$ because the higher level excited state of ${ }^{2} \mathrm{H}_{11 / 2}$ could receive more quenching effects. ${ }^{55}$ At elevated temperatures, not only the thermal factor of nanocrystals would become larger but also the surface quenching effect would be stronger. The slow increase rate of $I_{524} / I_{540}$ of core-shell nanocrystals in the low temperature range is due to the competition of the two different effects. Due to the inert shell which effectively blocks the quenching effect at high temperatures, the fast increasing rate of $I_{524} / I_{540}$ can attribute to the sole thermal factor. These data suggested that the coreshell UCNPs may not be sensitive for temperature measurements in the temperature range from $10{ }^{\circ} \mathrm{C}$ to $60{ }^{\circ} \mathrm{C}$.

Fig. 5(b) further shows that the ratios of $I_{540} / I_{655}$ emissions for the core and core-shell nanocrystals linearly decreased with temperature between $10{ }^{\circ} \mathrm{C}$ to $80{ }^{\circ} \mathrm{C}$, which was consistent with the results reported by other authors. ${ }^{57}$ Higher temperature increases the comparative influence of the non-radiative relaxation channels, from ${ }^{4} \mathrm{I}_{11 / 2}$ to ${ }^{4} \mathrm{I}_{13 / 2}$ and from ${ }^{4} \mathrm{~S}_{3 / 2},{ }^{2} \mathrm{H}_{11 / 2}$ to ${ }^{4} \mathrm{~F}_{9 / 2}$, leading to an increase of the ratio of $I_{540} / I_{655}$ emission.

\section{Conclusion}

In summary, we demonstrated here that oleic acid and oleylamine as co-surfactants with a right balance can be used to synthesize homogeneous core-shell $\mathrm{NaYF}_{4}$ nanocrystals. The intact shells with controlled thickness were useful in fully protecting the core nanocrystal from quenching by the surface ligands and solvent. This passivation effect by the intact shells enhanced the luminescence intensities particularly for violet emissions by a factor of up to 30 times. Our key finding reported here is that coating UCNPS with the inert shells improved the luminescent emission stability and reversibility of UCNPs against quenching caused by increased temperature or decreased $\mathrm{pH}$. Moreover, we found that the upconversion emissions at different temperatures for both core-only and core-shell UCNPs were fully recoverable, but 
once the UCNPs experienced lower $\mathrm{pH}$ conditions $(<\mathrm{pH}=7)$ their luminescence was observed to be permanently quenched suggesting that the acidic environment could chemically damage the surface of the nanocrystals. Furthermore, by a careful characterization, we found that the ratiometric emissions from the core-shell UCNPs were less sensitive to the response to the temperature changes in the range from $10{ }^{\circ} \mathrm{C}$ to $60{ }^{\circ} \mathrm{C}$. This work suggests that the luminescence stability and reversibility properties of UCNPs and their core-shell design should be taken into consideration for nanoscale bio-sensing applications in various temperature and $\mathrm{pH}$ conditions.

\section{Acknowledgements}

We thank D. Birch and Nicole in the Microscope Unit at Macquarie University for their help with TEM characterization. This project is primarily supported by the Australian Research Council (ARC) Future Fellowship Scheme (FT 130100517; D. Jin), Macquarie University Research Fellowship Scheme (X. Xu) and China Scholarship Council CSC scholarships (No. 201206170136, D. Liu). D. Jin and E. Goldys acknowledge partial support from the ARC Centre of Excellence for Nanoscale Biophotonics CE14010003.

\section{References}

1 B. Zhou, B. Y. Shi, D. Y. Jin and X. G. Liu, Nat. Nanotechnol., 2015, 10, 924-936.

2 D. Liu, X. Xu, Y. Du, X. Qin, Y. Zhang, C. Ma, S. Wen, W. Ren, E. M. Goldys, J. A. Piper, S. Dou, X. Liu and D. Jin, Nat. Commun., 2016, 7, 10254, DOI: 10.1038/ncomms10254. 3 F. Wang and X. Liu, J. Am. Chem. Soc., 2008, 130, 5642-5643. 4 Y. Lu, J. Zhao, R. Zhang, Y. Liu, D. Liu, E. M. Goldys, X. Yang, P. Xi, A. Sunna and J. Lu, Nat. Photonics, 2014, 8, 32-36.

5 J. B. Zhao, Z. D. Lu, Y. D. Yin, C. Mcrae, J. A. Piper, J. M. Dawes, D. Y. Jin and E. M. Goldys, Nanoscale, 2013, 5, 944-952.

6 J. Shen, L. Zhao and G. Han, Adv. Drug Delivery Rev., 2013, 65, 744-755.

7 S. Wu, G. Han, D. J. Milliron, S. Aloni, V. Altoe, D. V. Talapin, B. E. Cohen and P. J. Schuck, Proc. Natl. Acad. Sci. U. S. A., 2009, 106, 10917-10921.

8 D. Lu, S. K. Cho, S. Ahn, L. Brun, C. J. Summers and W. Park, ACS Nano, 2014, 8, 7780-7792.

9 A. Gnach, T. Lipinski, A. Bednarkiewicz, J. Rybka and J. A. Capobianco, Chem. Soc. Rev., 2015, 44, 1561-1584.

10 D. E. Achatz, R. Ali and O. S. Wolfbeis, Top. Curr. Chem., Springer, 2010, pp. 29-50.

11 D. E. Achatz, R. Ali and O. S. Wolfbeis, Top. Curr. Chem., 2011, vol. 300, pp. 29-50.

12 Q. Liu, J. J. Peng, L. N. Sun and F. Y. Li, ACS Nano, 2011, 5, 8040-8048.
13 R. Arppe, T. Näreoja, S. Nylund, L. Mattsson, S. Koho, J. M. Rosenholm, T. Soukka and M. Schäferling, Nanoscale, 2014, 6, 6837-6843.

14 K. L. Wong, G. L. Law, Y. Y. Yang and W. T. Wong, Adv. Mater., 2006, 18, 1051-1054.

15 L. Cheng, C. Wang and Z. Liu, Nanoscale, 2013, 5, 23-37.

16 W. Zheng, S. Y. Zhou, Z. Chen, P. Hu, Y. S. Liu, D. T. Tu, H. M. Zhu, R. F. Li, M. D. Huang and X. Y. Chen, Angew. Chem., Int. Ed., 2013, 52, 6671-6676.

17 Q. Liu, W. Feng, T. S. Yang, T. Yi and F. Y. Li, Nat. Protoc., 2013, 8, 2033-2044.

18 P. Huang, W. Zheng, S. Zhou, D. Tu, Z. Chen, H. Zhu, R. Li, E. Ma, M. Huang and X. Chen, Angew. Chem., Int. Ed., 2014, 53, 1252-1257.

19 Y. Min, J. Li, F. Liu, E. K. Yeow and B. Xing, Angew. Chem., Int. Ed., 2014, 53, 1012-1016.

20 Y. Yang, Q. Shao, R. Deng, C. Wang, X. Teng, K. Cheng, Z. Cheng, L. Huang, Z. Liu and X. Liu, Angew. Chem., Int. Ed., 2012, 51, 3125-3129.

21 X. Wu, Y. Zhang, K. Takle, O. Bilsel, Z. Li, H. Lee, Z. Zhang, D. Li, W. Fan and C. Duan, ACS Nano, 2016, 10, 1060-1066.

22 D. M. Yang, P. A. Ma, Z. Y. Hou, Z. Y. Cheng, C. X. Li and J. Lin, Chem. Soc. Rev., 2015, 44, 1416-1448.

23 Y. M. Yang, Microchim. Acta, 2014, 181, 263-294.

24 F. Vetrone, R. Naccache, A. Zamarron, A. Juarranz de la Fuente, F. Sanz-Rodríguez, L. Martinez Maestro, E. Martín Rodriguez, D. Jaque, J. García Solé and J. A. Capobianco, ACS Nano, 2010, 4, 3254-3258.

25 R. J. Meier, J. M. Simbürger, T. Soukka and M. Schäferling, Anal. Chem., 2014, 86, 5535-5540.

26 Y. L. Dai, P. A. Ma, Z. Y. Cheng, X. J. Kang, X. Zhang, Z. Y. Hou, C. X. Li, D. M. Yang, X. F. Zhai and J. Lin, ACS Nano, 2012, 6, 3327-3338.

27 Z. Y. Hou, C. X. Li, P. A. Ma, Z. Y. Cheng, X. J. Li, X. Zhang, Y. L. Dai, D. M. Yang, H. Z. Lian and J. Lin, Adv. Funct. Mater., 2012, 22, 2713-2722.

28 X. Zhang, P. P. Yang, Y. L. Dai, P. A. Ma, X. J. Li, Z. Y. Cheng, Z. Y. Hou, X. J. Kang, C. X. Li and J. Lin, Adv. Funct. Mater., 2013, 23, 4067-4078.

29 Y. L. Dai, D. M. Yang, P. A. Ma, X. J. Kang, X. Zhang, C. X. Li, Z. Y. Hou, Z. Y. Cheng and J. Lin, Biomaterials, 2012, 33, 8704-8713.

30 P. Zhao, J. Zhang, Y. H. Zhu, X. L. Yang, X. Jiang, Y. Yuan, C. S. Liu and C. Z. Li, J. Mater. Chem. B, 2014, 2, 8372-8377.

31 Y. Zhang, L. Huang, Z. Li, G. Ma, Y. Zhou and G. Han, ACS Nano, 2016, 10, 3881-3885.

32 N. Bogdan, F. Vetrone, G. A. Ozin and J. A. Capobianco, Nano Lett., 2011, 11, 835-840.

33 W. F. Xingjun Zhu, J. Chang, Y.-W. Tan, J. Li, M. Chen, Y. Sun and F. Li, Nat. Commun., 2016, 7, 10.

34 Y. Dai, P. a. Ma, Z. Cheng, X. Kang, X. Zhang, Z. Hou, C. Li, D. Yang, X. Zhai and J. Lin, ACS Nano, 2012, 6, 3327-3338.

35 E. E. Lees, T.-L. Nguyen, A. H. Clayton and P. Mulvaney, ACS Nano, 2009, 3, 1121-1128.

36 S. Wang, J. Feng, S. Song and H. Zhang, CrystEngComm, 2013, 15, 7142-7151. 
37 P. Sharma, S. Brown, G. Walter, S. Santra and B. Moudgil, Adv. Colloid Interface Sci., 2006, 123, 471-485.

38 Y. C. Wei, Q. Chen, B. Y. Wu, A. G. Zhou and D. Xing, Nanoscale, 2012, 4, 3901-3909.

39 D. D. Li, Q. Y. Shao, Y. Dong and J. Q. Jiang, Mater. Lett., 2013, 110, 233-236.

40 D. Li, Q. Shao, Y. Dong and J. Jiang, J. Phys. Chem. C, 2014, 118, 22807-22813.

41 B. Zhou, B. Shi, D. Jin and X. Liu, Nat. Nanotechnol., 2015, 10, 924-936.

42 J. C. Boyer and F. C. J. M. van Veggel, Nanoscale, 2010, 2, 1417-1419.

43 D. Q. Chen and P. Huang, Dalton Trans., 2014, 43, 11299-11304.

44 Y. F. Wang, L. D. Sun, J. W. Xiao, W. Feng, J. C. Zhou, J. Shen and C. H. Yan, Chem. - Eur. J., 2012, 18, 5558-5564.

45 N. Bogdan, E. M. Rodríguez, F. Sanz-Rodríguez, M. C. I. de la Cruz, Á. Juarranz, D. Jaque, J. G. Solé and J. A. Capobianco, Nanoscale, 2012, 4, 3647-3650.

46 N. J. J. Johnson, A. Korinek, C. H. Dong and F. C. J. M. van Veggel, J. Am. Chem. Soc., 2012, 134, 11068-11071.

47 L. X. Zhang, A. Mckay and D. Y. Jin, RSC Adv., 2013, 3, 8670-8673.

48 N. J. Johnson and F. C. van Veggel, Nano Res., 2013, 6, 547-561.
49 L. P. Qian, D. Yuan, G. S. Yi and G. M. Chow, J. Mater. Res., 2009, 24, 3559-3568.

50 Q. Tian, K. Tao and K. Sun, Micro Nano Lett., 2013, 8, 731-734.

51 L. X. Liu, F. Qin, H. Zhao, T. Q. Lv, Z. G. Zhang and W. W. Cao, Opt. Lett., 2013, 38, 2101-2103.

52 J. Shen, G. Y. Chen, T. Y. Ohulchanskyy, S. J. Kesseli, S. Buchholz, Z. P. Li, P. N. Prasad and G. Han, Small, 2013, 9, 3213-3217.

53 S. Zhou, W. Zheng, Z. Chen, D. Tu, Y. Liu, E. Ma, R. Li, H. Zhu, M. Huang and X. Chen, Angew. Chem., 2014, 126, 12706-12710.

54 J. Xu, S. Zhou, D. Tu, W. Zheng, P. Huang, R. Li, Z. Chen, M. Huang and X. Chen, Chem. Sci., 2016, 7, 2572-2578.

55 F. Wang, J. Wang and X. Liu, Angew. Chem., 2010, 122, 7618-7622.

56 L. H. Fischer, G. S. Harms and O. S. Wolfbeis, Angew. Chem., Int. Ed., 2011, 50, 4546-4551.

57 W. Yu, W. Xu, H. Song and S. Zhang, Dalton Trans., 2014, 43, 6139-6147.

58 Y. Tian, B. Tian, P. Huang, L. Wang and B. Chen, RSC Adv., 2015, 5, 14123-14128.

59 J. Xi, M. Ding, J. Dai, Y. Pan, D. Chen and Z. Ji, J. Mater. Sci.: Mater. Electron., 2016, 27, 8254-8270. 